\title{
NOVEL METHOD OF PROTECTING AND CONNECTING CMOS CHIPS ENABLES NETWORKED PACING LEADS
}

\author{
R. Azevedo, B. Costello, J. Frank, M. Jensen, T. Thompson, M. Zdeblick ${ }^{1}$ \\ ${ }^{1}$ Proteus Biomedical, Inc., Redwood City, California, USA
}

\section{BACKGROUND}

Implantable Pulse Generators (IPGs) are implantable, programmable devices intended to deliver electrical energy to body tissues over a period of five to ten years. This energy is typically delivered to the targeted tissue via an elongate lead. Cardiac leads, for example, are $\sim 80 \mathrm{~cm}$ long and are comprised of two or more insulated wires, each connected to a connector on the proximal end and an electrode ring on the distal end. In such systems, all of the electronics are in the IPG and the leads simply deliver energy. This architecture limits the number of electrodes and sensors available in a small form factor, and, thus has limited the clinical utility, cost-effectiveness and acceptance of implanted therapies.

A networked lead has enhanced functionality that is enabled by having active electronics embedded in the lead. In order to have commercial and clinical relevance, these electronics need to be protected from attack by body fluids and electrochemical reactions that result from delivering electrical energy to nearby tissue. Connections between the wires in the lead and the chip, and between the chip and the electrodes, must be made in a way that is robust and protected from corrosion and fatigue.

MEMS discipline offers unique planar methods of both protecting the CMOS chip that is embedded in the lead and providing means to connect to it in a robust fashion. This work describes this Chipskin ${ }^{\mathrm{TM}}$ technology applied to a Networked Lead fpr application in cardiac rhythm management.

\section{EXPERIMENTAL SETUP}

An adaptive pacing system is comprised of a Networked Lead and an IPG capable of communicating with devices on the lead. The Networked Lead, in turn, is comprised of a standard IS-1 connector on one end and a number of "satellites" on the other. Inside each satellite is a Chipskin protected CMOS chip connected to two wires and four electrodes (Figures $1 \& 2$ ).

\section{Networked Lead}

A Networked Lead is capable of receiving communication signals from a central controller using a duplex communication system. As shown in Figure 1, two wires are used to communicate with four "satellites" near the distal end of the lead, each satellite supporting four addressable electrodes.

The primary commands associated with this device include: Clear, Switch (or configure), Talkback, and Sleep. The latter puts the satellite in a state where it is directing energy while consuming less than $10 \mathrm{pA}$. These commands are communicated by impressing a high-frequency pattern on a pace pulse: the satellite scavenges the energy from the pace pulse, interprets the command, and executes the instruction if its address matches. Talkback is a command used to confirm the configuration by a given satellite.

Shown in Figure 2 is a Finite Element Analysis with the voltage distribution surrounding these electrodes during a pacing pulse, and the radially-specific distribution that is enabled by this networked lead design.. In one application for cardiac resynchronization therapy, radially-selective pacing is used to mitigate the "capture" of the nearby phrenic nerve at the most preferred pacing site. Phrenic nerve capture is a common sideeffect of pacing from a cardiac vein, reported by patients as a "hiccup" occurring once per second. Current leads with two or four hard-wired electrodes allow electronic repositioning, but at the expense of pacing site location, which is a critical factor in optimizing clinical outcome.

The use of smaller electrodes also reduces the amount of energy required to capture cardiac tissue, and thus increases battery life. In addition to radially-selective pacing, the same networked lead can increase both the volume and fidelity of electrical field sensing. For example, by switching from a dipole on one satellite to a dipole on another satellite, it is possible to measure the time it takes for a depolarization wave to travel from one satellite to another. This multi-dimensional information may be helpful in titrating dosage of certain anti-arrhythmia drugs, such as Amiodarone.

\section{Chipskin Technology \& Satellite Construction}

Each Chipskin satellite is welded to four electrodes. The satellite is comprised of a CMOS silicon chip protected with Chipskin technology that is attached to a Pt/Ir chip carrier and potted in epoxy.

The Chipskin technology provides two important and innovative functions: first, it provides a corrosion-resistant, hermetic package that protects the silicon chip and its interconnects from corrosion in very harsh environments. Second, it provides a method for integrated and weldable interconnects, assuring robust mechanical and electrical connections from the IC to the conductors and the electrodes. The cross section shown in Figure 3 depicts a cantilever of a thick, corrosion-resistant $\mathrm{Pt} / \mathrm{Ir}$ alloy. Figure 4 shows a micrograph of an early-model chip illustrating the cantilevered connectors.

The Chipskin process starts with a complete CMOS wafer. In this work, ON Semiconductor's C5 process was used. In the first step the wafer is coated with a series of corrosion-resistant dielectric coatings and metals, including SiC, Ir, PtIr, etc. These coatings have been characterized for corrosion inside the body by implanting them in animals for extended periods and measuring the change in thickness, with and without pacing occurring nearby. From this, one can determine the amount of dissolution that may be expected over ten years, which is a small fraction of the thickness of the films. Next, a thin layer of Pt is deposited and patterned. This is followed by a second layer of the dielectric, which is deposited and patterned. Next, a thick layer of Pt/Ir is deposited using a custom, vacuum-based physical deposition system. This produces a low stress layer of $\mathrm{Pt} / \mathrm{Ir}$ that is between 50 and 75 um thick. Then, the back surface of the silicon wafer is patterned and etched using Reactive Ion Etching. This leaves the bulk of the chip suspended from the wafer by the thick Pt/Ir beams. The backside of the chip is then coated with one or more of a number of different protection materials, including, for example, $\mathrm{TaO}, \mathrm{TiO}$, and $\mathrm{SiC}$, which completely surround the chip and cantilever beams. This effectively puts a protective IPG "can" on the chip using planar-processed feedthroughs to connect the electronics to its package. The cantilevers are thick enough to support the stress of welding during connection to surrounding $\mathrm{Pt} / \mathrm{Ir}$ structures and are designed and tested to withstand over $400 \mathrm{M}$ cycles of bending typically seen by a lead in the heart over a ten year period. 


\section{RESULTS}

The described leads made with the construction techniques described above have been subjected to the equivalent of over 50 years of pacing in vitro and over one year in vivo. These and other testing results confirm that this technique is robust against the corrosion expected during a ten year period of cardiac pacing.

In addition, as shown in Figure 5, the networked lead has been shown to eliminate phrenic nerve capture at the ideal pacing site location. This pre-clinical study compared conventional lead pacing in the "band-to-band" and "band-to-coil" configurations versus the radially-selective networked lead pacing, using "intraband" configurations, i.e., from one electrode on a satellite to the pair of electrodes on either side of that electrode on the same satellite. While conventional leads, which feature axially symmetric pacing electrodes capture the phrenic nerve at clinically unacceptable low voltages (between 4 and 5 volts), identical electrodes in identical positions using the networked lead programmed for intraband pacing do not capture the phrenic nerve even at $18 \mathrm{~V}$ pacing.

\section{CONCLUSIONS AND RECOMMENDATIONS}

Protecting a silicon chip from corrosion within a Networked Lead has been shown to be possible using in vitro and in vivo studies. In addition, this packaging and connection technology expands and enables new applications for distributed electronics and sensors, particularly in environments where harsh conditions, size, cost and durability of tags or sensors it critical. Examples include marine chemistry, petroleum exploration, and portable electronics that need to be robust against exposure or immersion in water or other corrosive environments.

The use of Networked Leads is likely to expand from cardiac applications to neural pacing for pain, epilepsy, Parkinson's, hearing loss, and other ailments. While this generation is focused on the multiplex function, future devices are likely to add sophisticated analog sensing circuitry applied at the point of interest and technology to improve the procedure reliability of pulse-delivering leads. For example, it is likely that circuitry that protects the body from currents in lead wires exposed to MRI fields will be perfected, allowing cardiac pacemaker patients to benefit from MRI imaging anywhere in their body.

Distributing electronics away from a central computer created internet computing; networking electronics in vivo may lead to a similar generation of innovation of sensing, computing and communicating from the skin ... in

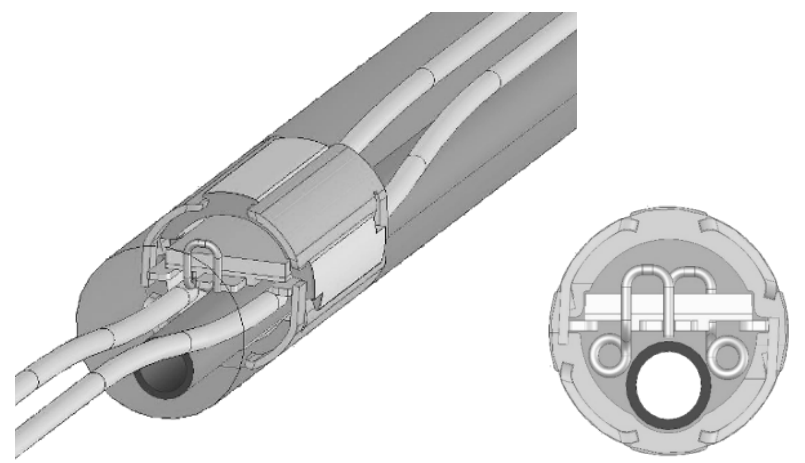

Figure 1: CAD views of silicon chip between lead wires and electrodes

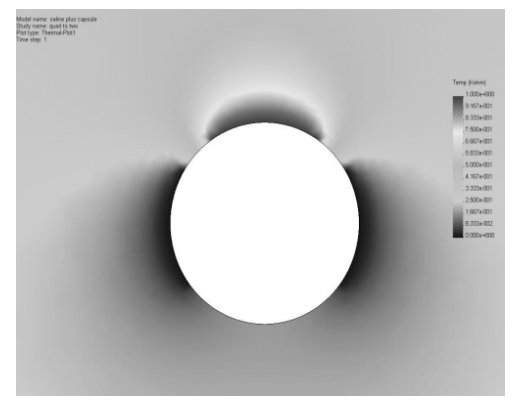

Figure 2: Finite element result of radially-selective (intra-band) pacing

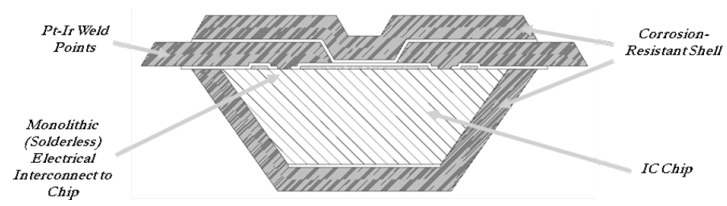

Figure 3: Cross section of a Chipskin ${ }^{\mathrm{TM}}$ protected silicon chip

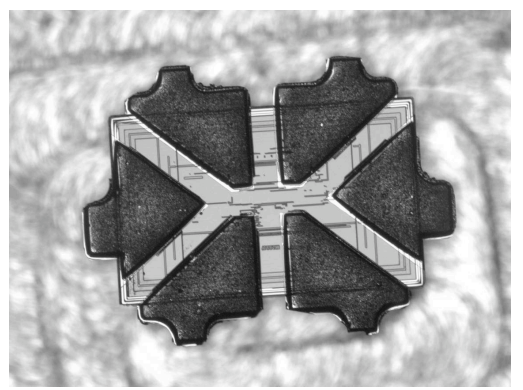

Figure 4: Microphotograph of a Chipskin ${ }^{\mathrm{TM}}$ protected silicon chip

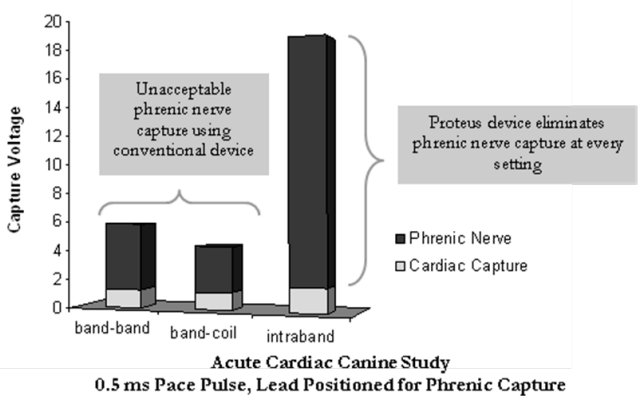

Figure 5: In-vivo cardiac model demonstration of radiallyselective (intra-band) pacing using a Networked Lead to eliminate phrenic nerve capture vs. conventional leads 